\title{
General Criterion for Harmonicity
}

\author{
Karel Proesmans* and Hans Vandebroek \\ Hasselt University, B-3590 Diepenbeek, Belgium \\ Christian Van den Broeck \\ Hasselt University, B-3590 Diepenbeek, Belgium, Stellenbosch Institute of Advanced Studies, Matieland 7602, South Africa
}

(Received 3 March 2017; published 6 October 2017)

\begin{abstract}
Inspired by Kubo-Anderson Markov processes, we introduce a new class of transfer matrices whose largest eigenvalue is determined by a simple explicit algebraic equation. Applications include the free energy calculation for various equilibrium systems and a general criterion for perfect harmonicity, i.e., a free energy that is exactly quadratic in the external field. As an illustration, we construct a "perfect spring," namely, a polymer with non-Gaussian, exponentially distributed subunits which, nevertheless, remains harmonic until it is fully stretched. This surprising discovery is confirmed by Monte Carlo and Langevin simulations.
\end{abstract}

DOI: 10.1103/PhysRevLett.119.147803

The stretching of an (ideal) polymer provides one of the most beautiful illustrations of thermodynamics and equilibrium statistical physics. A force is needed because the number of polymer configurations corresponding to a stretched state is (exponentially) smaller than that of a coiled state. More generally, the extension $X$ versus force $F$ relation is obtained from the derivative, at constant temperature $T$, of the free energy $\mathcal{G}$ [1]

$$
X=-d \mathcal{G} / d F \text {. }
$$

Under isotropic conditions, one expects that the free energy $\mathcal{G}$ is even in $F$, hence, quadratic for $F$ small. The extension $X$ is then harmonic, i.e., linear in the force $F$. For larger forces, the polymer is expected to stiffen as it approaches full extension. For example, for a freely jointed chain in three dimensions, consisting of $N$ units of fixed length $b$, the fractional extension $x=X / N b$ is given by $x=\mathcal{L}(b F \beta)$ with $\mathcal{L}$ the famous Langevin function $\mathcal{L}(y)=\operatorname{coth}(y)-$ $1 / y[2]$ and $\beta=1 /\left(k_{B} T\right)$, see, also, Fig. 1(a).

The issue of elasticity is an important one in materials science. The width of the (harmonic) elastic regime, which can be estimated from the ratio of the tensile strength over the Young's modulus, varies greatly from very small, for example for nanotubes, to very large for rubber and elastin [3]. Therefore, it is natural to ask whether variations of a basic microscopic model, such as the freely jointed chain, can lead to a predominant or even perfect harmonic response. The immediate answer appears to be yes: the Rouse model [4] supposes bonds that are perfectly harmonic, and hence, so is the entire chain; but the assumption of perfectly harmonic bonds is unphysical as it would, for example, imply that both the bond and chain can be infinitely stretched, and deriving harmonicity from harmonicity is not exactly a great feat. The surprising finding of this Letter is the discovery of a simple random walk model for a polymer with nonharmonic bonds which is and remains perfectly harmonic up to full extension (and not beyond).

Before proceeding to the more technical derivation, we comment on the route that led to this discovery and the additional results that were obtained. The statistical physics literature on polymers is huge, but exact results can only be derived for some very simple models such as the freely jointed chain $[5,6]$. One of the main tools for arriving at these results is the evaluation of the partition function via a transfer matrix method, essentially by identifying the largest eigenvalue. Such transfer matrices have positive entries and are, therefore, reminiscent of Markov matrices, which describe the dynamics of Markov chains. We introduce a special class of transfer matrices whose structure is inspired by a specific type of Markov process. We coin the name "Kubo-Anderson" transfer matrices in reference to two early papers (on linewidth problems) in which such Markov processes have been introduced [7]. The bonus is that the largest eigenvalue of such a transfer matrix is determined by a simple explicit algebraic equation. Applications include the free energy calculation for various equilibrium systems, including a simple model for polymer chains with persistence. Furthermore, we derive, after an additional simplification, a general criterion for perfect harmonicity, i.e., a free energy that is exactly quadratic in the external field. We will discuss it here in the context of a simple random walk model for a polymer, but the results are equally valid for other systems, such as magnetic systems with exactly linear susceptibility. The application to simple polymer models leads to the discovery of the "perfect spring," i.e., a random walk model for a polymer with non-Gaussian subunits which, nevertheless, remains harmonic until it is fully stretched. 


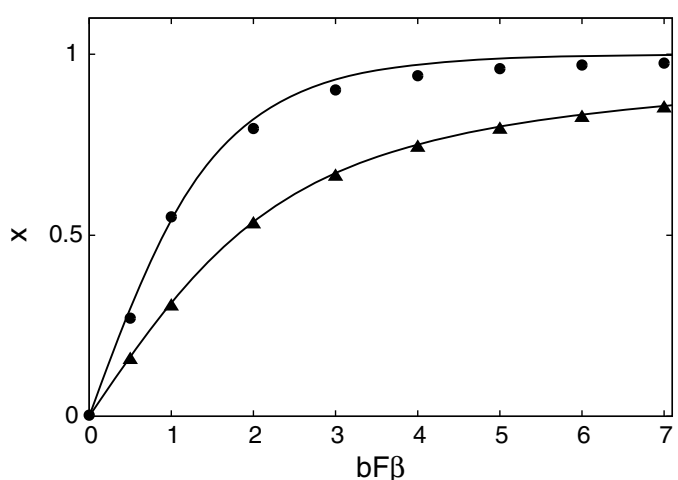

(a)

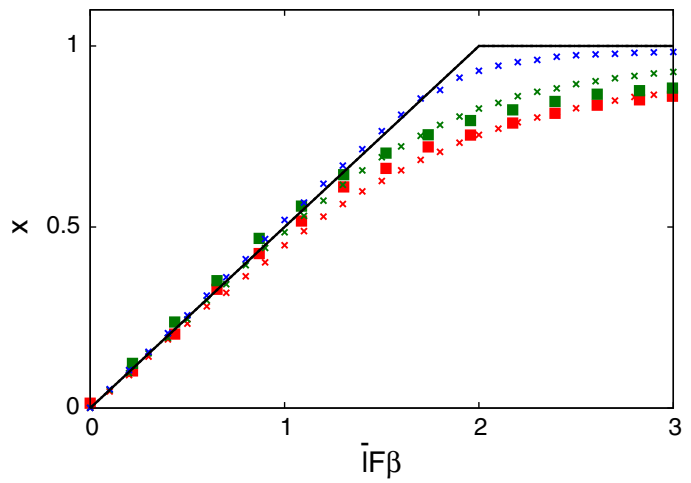

(b)

FIG. 1. Extension $x$ versus force $F$ for (a) $d=3$ freely jointed chain $q=1$ and its modified version with persistence $q=0.7$ : theoretical result Eq. (16) (solid line) versus Langevin simulation for $N=100$ (triangles $q=1$ and dots $q=0.7$ ). (b) Random walk polymer model with persistence and appropriate transverse field reproducing Eq. (28). $L=10 \mathrm{dl}$ (red), $L=20 \mathrm{dl}$ (green), and $L=100 d l$ (blue), with $\bar{l} / d l=10$ (Monte Carlo, crosses) and $\bar{l} / d l=5$ (Langevin, squares).

As is well known [8], many problems in equilibrium statistical mechanics, including the celebrated Onsager solution of the two-dimensional Ising model, can be formulated in terms of a transfer matrix. One supposes that the energy of the system can be written as a sum $E=\sum_{i=1}^{N} E_{\sigma_{i+1}, \sigma_{i}}$, where $\sigma_{i}$ represents the state of the "ith layer" and $E_{\sigma_{i+1}, \sigma_{i}}$ is the interaction energy between layers $i+1$ and $i$. For notational simplicity, we consider periodic boundary conditions with layer $N+1$ identified with layer 1. The central quantity is the partition function

$$
Z=\sum_{\{\sigma\}} e^{-\beta \sum_{i=1}^{N} E_{\sigma_{i+1}, \sigma_{i}}}
$$

where the sum over $\{\sigma\}$ runs over all configurational states of the system. The transfer matrix $\mathbf{T}$ is defined by its elements

$$
\mathbf{T}_{\sigma^{\prime}, \sigma}=e^{-\beta E_{\sigma^{\prime}, \sigma}} .
$$

In the thermodynamic limit $N \rightarrow \infty$, the free energy $\mathcal{G}$ is obtained as

$$
\mathcal{G}=-\frac{\ln Z}{\beta}=-\frac{\operatorname{Tr} \mathbf{T}^{N}}{\beta} \sim-\frac{N \ln \lambda}{\beta},
$$

where $\sim$ refers to an equality to dominant order in $N$, and $\lambda$ is the largest eigenvalue of the transfer matrix $\mathbf{T}$.

A transfer matrix has positive elements. The elements of a Markov matrix represent probabilities, which are also obviously positive but obey, in addition, a normalization condition. The relation between transfer matrices and Markov matrices was noticed a long time ago, see, e.g., $[9,10]$, and has been revisited more recently in the context of large deviations for conditioned Markov processes [11]. In this Letter, we introduce a new class of transfer matrices inspired by Kubo-Anderson and Markov processes. The latter have been used for a detailed analytic description of a large variety of physical processes [7]. In the context of transfer matrices, the bonus is a simple explicit relation for its largest eigenvalue. Our starting point is the following Markov matrix:

$$
\mathbf{A}_{\sigma^{\prime}, \sigma}=\left(1-q_{\sigma}\right) \delta_{\sigma, \sigma^{\prime}}+q_{\sigma} p_{\sigma^{\prime}} .
$$

$\mathbf{A}_{\sigma^{\prime}, \sigma}$ is the transition probability to go from state $\sigma$ to $\sigma^{\prime}$. Its explicit form can be explained as follows. With a probability $1-q_{\sigma}$ the system remains in its present state $\sigma$. With probability $q_{\sigma}$, a novel state is selected. This novel state is $\sigma^{\prime}$ with probability $p_{\sigma^{\prime}}$. As is explained in the introduction, with the stretching of a polymer, the addition of an external field allows us to explore the regions of "higher free energy." We do something similar here by adding an extra Boltzmann factor to the transfer matrix, with $\epsilon_{\sigma}$ representing the energy contribution due to such a field on layer $\sigma$, leading finally to the following Kubo-Anderson transfer matrix:

$$
\mathbf{T}_{\sigma^{\prime}, \sigma}=e^{-\frac{\beta\left(\sigma_{\sigma}+\epsilon_{\sigma^{\prime}}\right)}{2}} \mathbf{A}_{\sigma^{\prime}, \sigma} .
$$

The connection with the corresponding interaction energy $E_{\sigma^{\prime}, \sigma}$ is obtained by comparison with Eq. (3). Conversely, this relation establishes the dependence of the quantities $q_{\sigma}$ and $p_{\sigma}$ on the energies $E_{\sigma^{\prime}, \sigma}$ and the temperature.

We now show how the largest eigenvalue (and corresponding eigenfunction) of a Kubo-Anderson transfer matrix $T$ can be obtained. From the eigenvalue equation

$$
\sum_{\sigma} \mathbf{T}_{\sigma^{\prime}, \sigma} \phi_{\sigma}=\lambda \phi_{\sigma^{\prime}}
$$

one finds that

$$
e^{-\beta \epsilon_{\sigma^{\prime}}}\left(1-q_{\sigma^{\prime}}\right) \phi_{\sigma^{\prime}}+p_{\sigma^{\prime}} \sum_{\sigma} e^{-\frac{\beta\left(\epsilon_{\sigma}+\epsilon_{\sigma^{\prime}}\right)}{2}} q_{\sigma} \phi_{\sigma}=\lambda \phi_{\sigma^{\prime}} .
$$


The largest eigenvalue $\lambda$ and its corresponding eigenfunction $\phi$ can be identified by invoking the Perron-Frobenius theorem: the eigenvalue $\lambda$ is unique and positive and all components of $\phi$ have the same sign, and can, thus, be chosen to be positive. As a consequence $\sum_{\sigma} \exp \left(-\beta \epsilon_{\sigma} / 2\right) q_{\sigma} \phi_{\sigma}$ is also positive. Furthermore, since $\phi$ is only determined up to a constant factor, we can assume the following normalization:

$$
\sum_{\sigma} e^{-\frac{\beta \epsilon_{\sigma}}{2}} q_{\sigma} \phi_{\sigma}=1
$$

With this constraint, we find, from Eq. (8), the following explicit expression for the eigenvector $\phi$ :

$$
\phi_{\sigma}=\frac{p_{\sigma}}{\lambda e^{\frac{\beta \epsilon_{\sigma}}{2}}-e^{-\frac{\beta \epsilon_{\sigma}}{2}}\left(1-q_{\sigma}\right)} .
$$

By substitution of this expression in Eq. (9), one concludes that $\lambda$ is determined by

$$
\sum_{\sigma} \frac{p_{\sigma} q_{\sigma}}{e^{\beta \epsilon_{\sigma}} \lambda-\left(1-q_{\sigma}\right)}=1
$$

This simple explicit algebraic equation for the dominant eigenvalue is the first important result of this Letter.

We mention a few classes of systems which can be solved exactly. As a first example, we consider systems without "persistence," i.e., $q_{\sigma} \equiv 1$, leading to

$$
\lambda=\sum_{\sigma} p_{\sigma} e^{-\beta \epsilon_{\sigma}} .
$$

The 3D freely jointed chain is obtained with the following identifications: $\sigma$ represents the space angle $\boldsymbol{\Omega}$, specifying the orientation of each subunit (of fixed length $b$ ). Since this orientation is random, one has $p_{\Omega}=1 /(4 \pi)$, while the sum becomes an integral over the space angle, $\sum_{\sigma} \rightarrow \int_{0}^{2 \pi} d \phi \int_{0}^{\pi} d \theta \sin \theta$. Furthermore, $\epsilon_{\boldsymbol{\Omega}}=F b \cos \theta$ represents the effect of an external field, with $\theta$ the angle between the bond and this field. Equation (12) gives the result

$$
\lambda=\frac{\sinh (b F \beta)}{b F \beta} .
$$

By combination with Eq. (1) and Eq. (4), one recovers the aforementioned result $x=X /(N b)=\mathcal{L}(b F \beta)$.

The above analysis can be reproduced for state-independent persistence, i.e., $q \leq 1$ but independent of $\sigma=\boldsymbol{\Omega}$, by starting from Eq. (11) rather than from Eq. (12). The integral determining the eigenvalue $\lambda$

$$
\int_{0}^{2 \pi} d \phi \int_{0}^{\pi} d \theta \frac{\sin \theta}{4 \pi} \frac{q}{e^{b F \beta \cos \theta} \lambda-(1-q)}=1,
$$

can still be solved

$$
\lambda=\frac{\left(e^{2 b F \beta / q}-1\right)(1-q)}{e^{b F \beta(2 / q-1)}-e^{b F \beta}} .
$$

The fractional extension $x=\mathcal{L}_{q}(b F \beta)$ is described by a generalized Langevin function, see, also, Fig. 1(a),

$$
\mathcal{L}_{q}(y)=\frac{(2-q) e^{2 y / q}\left(e^{2 y}-1\right)+q\left(e^{2 y}-e^{4 y / q}\right)}{q\left(e^{2 y / q}-1\right)\left(e^{2 y}-e^{2 y / q}\right)} .
$$

The freely jointed chain is retrieved in the limit $q \rightarrow 1$, with $\mathcal{L}_{q}$ converging to $\mathcal{L}$. In the limit of strong persistence, $q \rightarrow 0, \mathcal{L}_{q}$ converges to the sign function $\mathcal{L}_{0}(y)=\operatorname{sgn}(y)$. For small forces, the polymer behaves as a harmonic spring, with spring constant $\kappa_{q}=F / X$ given by

$$
\kappa_{q}=\frac{q}{(2-q)} \kappa_{1} \quad \text { with } \quad \kappa_{1}=\frac{3}{N b^{2} \beta} .
$$

$\kappa_{1}$ is the spring constant of the $3 \mathrm{D}$ freely jointed chain. Note the weakening of the spring for increasing persistence, corresponding to a decreasing value of $q$. These predictions have been verified using Langevin simulations, cf. Fig. 1(a). Other applications, e.g., 1D polymers and systems with only two $\sigma$ states, are presented in the Supplemental Material [12].

Depending on the interpretation of the model, the above transfer matrix describes discrete steps taking place in space (for example, in an Ising spin or polymer chain problem), in time (for a Markov chain), or in another possibly more abstract coordinate (for example, an angle coordinate or a higher dimensional vectorial coordinate). To further simplify the eigenvalue equation, we focus on a "hydrodynamic" limit. For simplicity, we present it as taking place in the context of a single scalar spatial variable. We associate an elementary spatial displacement of length $d l$ (corresponding to the bond length $b$ in the above polymer problem) to each discrete step. This length will be small compared to the average length of "straight" segments, belonging to a given state $\sigma, \bar{l}_{\sigma}$, i.e., $d l / \bar{l}_{\sigma} \rightarrow 0$. Meanwhile, the total length $L=N d l$ will become large compared to the typical lengths of the problem, $\bar{l}_{\sigma} / L \rightarrow 0$. In this limit, the "jump probabilities" $q_{\sigma}$ are replaced by transition probabilities per unit length $k_{\sigma}=1 / \bar{l}_{\sigma}$

$$
q_{\sigma}=k_{\sigma} d l,
$$

resulting in straight segments with lengths that are exponentially distributed. The matrix $\mathbf{A}$ is replaced by a transition matrix $\mathbf{K}$

$$
\mathbf{A}_{\sigma^{\prime}, \sigma}=\mathbf{1}+\mathbf{K}_{\sigma^{\prime}, \sigma} d l, \quad \mathbf{K}_{\sigma^{\prime}, \sigma}=k_{\sigma}\left(p_{\sigma^{\prime}}-\delta_{\sigma, \sigma^{\prime}}\right) .
$$

Consistent with this limit, the energy $\epsilon_{\sigma}$ and the eigenvalue $\lambda$ converge as follows to 0 and 1 , respectively, 


$$
\epsilon_{\sigma}=F_{\sigma} d l, \quad \lambda=1+\mu d l,
$$

with $F_{\sigma}$ representing the external "force" or "energy density" when the system is in the state $\sigma$. Thus, the eigenvalue equation, Eq. (11), reduces to the following algebraic relation for $\mu$ :

$$
\sum_{\sigma} \frac{p_{\sigma} k_{\sigma}}{\mu+\beta F_{\sigma}+k_{\sigma}}=1
$$

We note that the dependence on the external field contribution $F_{\sigma}$ is no longer via an exponential function. The corresponding free energy, cf. Eq. (4), becomes

$$
\mathcal{G} \sim-\frac{L \mu}{\beta},
$$

with a simple proportionality to the eigenvalue $\mu$. The extensivity in $N$ is replaced by an extensivity in $L$. Equation (22) is an equality to dominant order in $L$.

We now turn to the search for a perfect spring, defined as a system for which the free energy Eq. (22) is exactly quadratic in the external field amplitude. More precisely, we introduce the overall force $F$ via the specification $F_{\sigma}=a_{\sigma} F$, with $a_{\sigma}$ an $F$-independent amplitude, and require that

$$
\mu=l(\beta F)^{2},
$$

with $l$ an $F$-independent reference length scale. Thus, the question reduces to finding probability distributions $p_{\sigma}$ such that Eq. (21) holds under this constraint. By Taylor expansion in $F$, and under the assumption that $k_{\sigma}=1 / \bar{l}$ is independent of the state $\sigma$, one finds the following explicit expression for the moment generating function associated with $p_{\sigma}$ (see Supplemental Material [12])

$$
\sum_{\sigma} p_{\sigma} e^{-\lambda a_{\sigma}}=\sqrt{\frac{\bar{l}}{l}} \frac{I_{1}\left(2 \sqrt{\frac{l}{\bar{l}}} \lambda\right)}{\lambda} .
$$

$I_{1}$ is the modified Bessel function. This general criterion for harmonicity is our second major result. The probability distribution $p_{\sigma}$ can be found from it, depending on the topology of the phase space and the form of $a_{\sigma}$, by an inverse (integral) transform. Equation (24) obviously has no solution for a finite state space of $\sigma$. In particular, twostate models (corresponding, for example, to a polymer model in $d=1$ ) can not be turned into fully harmonic springs.

Combining Eq. (1), Eq. (22), and Eq. (23), one finds that the corresponding stretching fraction $x$ is given by

$$
x=X / L=-\frac{1}{L} \frac{d}{d F} \mathcal{G}=2 l F \beta, \quad x \leq 1 .
$$

Thus, the extension is exactly linear in $F$. The above result is only valid up to $x=1$, i.e., until the polymer is fully stretched. The reason for this limitation is that the Taylor expansion of Eq. (21) has a radius of convergence given by $F_{c}=1 /(2 l \beta)$. For larger values of $F, x$ stays put at its maximal value $x=1$; hence, the system undergoes a second order phase transition at $F=F_{c}$ (discontinuous second derivative of $\mathcal{G}$ ). The spring constant $\kappa$, corresponding to the harmonic law Eq. (25), is given by $\kappa=1 /(2 L l \beta)$. The above result has been derived in the limit $d l / \bar{l} \rightarrow 0$. In the Supplemental Material [12], we evaluate the first order correction in $d l$ and conclude that the harmonic behavior prevails, but with a modified spring constant

$$
\kappa=\frac{1}{2 L l \beta}\left(1-2 \frac{d l}{\bar{l}}\right)
$$

As a concrete application of the above harmonicity criterion, we return to the polymer problem in $d$-dimensional Euclidean space, with the identification of $\sigma$ as a $d$ dimensional spatial angle $\boldsymbol{\Omega}$. Identifying $b$ with $d l$, one finds, from $\epsilon_{\boldsymbol{\Omega}}=F b \cos \theta$, that $a_{\boldsymbol{\Omega}}=\cos \theta$. Furthermore, if we assume that $p_{\Omega}$ only depends on $\theta$, the integral Eq. (24) can be solved by inverting the integral transform. Anticipating that $l=\bar{l} / 4$ and comparing the harmonicity criterion, Eq. (24), with the following integral representation of the Bessel function [13]:

$$
I_{1}(\lambda)=\frac{\lambda}{\pi} \int_{0}^{\pi} d \theta e^{-\lambda \cos \theta} \sin ^{2} \theta,
$$

we conclude (remembering that the Jacobian of the $d$ sphere features the factor $\sin ^{d-2} \theta$ )

$$
p_{\Omega}=\mathcal{N}_{d} \sin ^{4-d} \theta .
$$

$\left(\mathcal{N}_{d}\right.$ is a normalization constant.) This is our third major result. We conclude that a polymer with persistence, consisting of exponentially distributed straight segments, is perfectly harmonic until full stretching in $d=4$. In $d=3$, one needs an additional field, orthogonal to the stretching direction, which induces a biased distribution $p_{\Omega} \sim \sin \theta$. Such a field can be realized by application of an electromagnetic force, which is often used in the experimental stretching of polymers. We have verified the latter prediction via Monte Carlo and Langevin simulations. The numerical results are in perfect agreement with the theory, cf. Fig. 1(b) and the Supplemental Material [12] for more details [14]. These conclusions are, of course, not restricted to polymer models.

In conclusion, we have introduced Kubo-Anderson transfer matrices, for which the largest eigenvalue can be obtained from the simple, explicit algebraic equation, Eq. (11). The latter simplifies, upon taking a continuous limit, to Eq. (21). By assuming a uniform transition rate, a 
simple, explicit criterion for harmonicity results, cf. Eq. (24). As a concrete example, we show that a polymer chain with persistence can behave, until fully stretched, as a perfect harmonic spring. Although our model is rather theoretical, and perhaps not experimentally feasible, its discovery can serve as the starting point for the construction of complex polymer systems with enhanced harmonicity. These results can be easily mapped on other systems. For example, one could construct an Ising-like magnet with perfectly linear susceptibility.

*Karel.Proesmans@uhasselt.be

[1] $\mathcal{G}=U-T S-F X$, with $U$ the internal energy and $S$ the entropy.

[2] W. Kuhn and F. Grun, Kolloid Z. 101, 248 (1942).

[3] J. Howard et al., Mechanics of Motor Proteins and the Cytoskeleton (Sinauer Associates, Sunderland, MA, 2001).

[4] P. E. Rouse, Jr, J. Chem. Phys. 21, 1272 (1953).

[5] P. Flory, M. Volkenstein et al., Statistical Mechanics of Chain Molecules (Wiley Online Library, New York, 1969).

[6] T. M. Birshtein, Conformations of Macromolecules (Interscience Publishers, New York, 1966).
[7] R. Kubo, J. Phys. Soc. Jpn. 9, 935 (1954); P. W. Anderson, J. Phys. Soc. Jpn. 9, 316 (1954); P. L. Bhatnagar, E. P. Gross, and M. Krook, Phys. Rev. 94, 511 (1954); P. Welander, Ark. Fys. 7, 507 (1954); H. G. Othmer, S. R. Dunbar, and W. Alt, J. Math. Biol. 26, 263 (1988); H. Dekker, G. de Leeuw, and A. Maassen van den Brink, Phys. Rev. E 52, 2549 (1995); A. Kaminska and T. Srokowski, Phys. Rev. E 67, 061114 (2003); 69, 062103 (2004); R. D.White, R. E. Robson, S. Dujko, P. Nicoletopoulos, and B. Li, J. Phys. D 42, 194001 (2009); C. Van den Broeck and R. Toral, Phys. Rev. E 89, 062124 (2014).

[8] R. J. Baxter, Exactly Solved Models in Statistical Mechanics (Dover Publications, Mineola, NY, 2007).

[9] H. Miller, Ann. Math. Stat. 32, 1260 (1961).

[10] M. E. Fisher, Am. J. Phys. 32, 343 (1964).

[11] R. Chetrite and H. Touchette, Ann. Inst. Henri Poincaré 16, 2005 (2015).

[12] See Supplemental Material at http://link.aps.org/ supplemental/10.1103/PhysRevLett.119.147803 for the details of calculations and simulations, and for extra examples.

[13] E. W. Weisstein, CRC Concise Encyclopedia of Mathematics (CRC Press, Boca Raton, FL, 2002).

[14] A. C. Brańka and D. M. Heyes, Phys. Rev. E 60, 2381 (1999). 